\title{
No Fault Compensation in der medizinischen Haftpflicht
}

Richard O. Binswanger

Interaktiver
Artikel
Wollen Sie diesen Artikel
kommentieren? Nutzen
Sie dafür die Kommentar-
funktion in der Online-
Version oder sehen Sie
nach, was Ihre Kolleginnen
und Kollegen bereits
geschrieben haben:
www.saez.ch/
aktuelle-ausgabe/
interaktive-beitraege/

Korrespondenz:

Dr. med. Richard O. Binswanger Facharzt für Radiologie und Nuklearmedizin

Führungsschule Bodensee Münsterlingen

Oberer Seeweg 9

CH-8597 Landschlacht

r.binswanger[at]bluewin.ch

www.fsb-spital.ch
An einem Kongress in Oxford berichtete der Chefarzt für Radiologie der Universitätsklinik Kopenhagen über die Nephrogene Systemische Sklerose, eine zum Teil tödliche Komplikation von MRI-Kontrastmitteln bei Nierenversagen. Diese Erkrankung war dort besonders häufig vorgekommen und schliesslich auch entdeckt worden. Denn auf Empfehlung u.a. der Radiologen wurden die Kontrastmittel besonders oft bei Niereninsuffizienz angewendet. Das war eine Katastrophe. Es gab einen Sturm, in dessen Auge sich der Radiologe befand. Eindrücklich beschrieb er am Kongress das Leiden der Patienten und das Geschehen von der Vermutung bis zur Gewissheit. Auf meine Frage, wie die Haftpflichtfrage gelöst worden sei, stellte sich heraus, dass alle Opfer in angemessener Höhe, rasch und anstandslos entschädigt wurden, ohne Frage nach dem Verschulden. Dieses System heisst No Fault Compensation. Es gilt neben Dänemark unter anderem auch in Neuseeland [1].

\section{Die Verhältnisse in der Schweiz}

Wenn ein Autofahrer ein Kind anfährt, kommt in der Schweiz die No Fault Compensation zur Anwendung. Die Haftpflichtversicherung des Lenkers übernimmt anstandslos den Schaden, auch wenn der

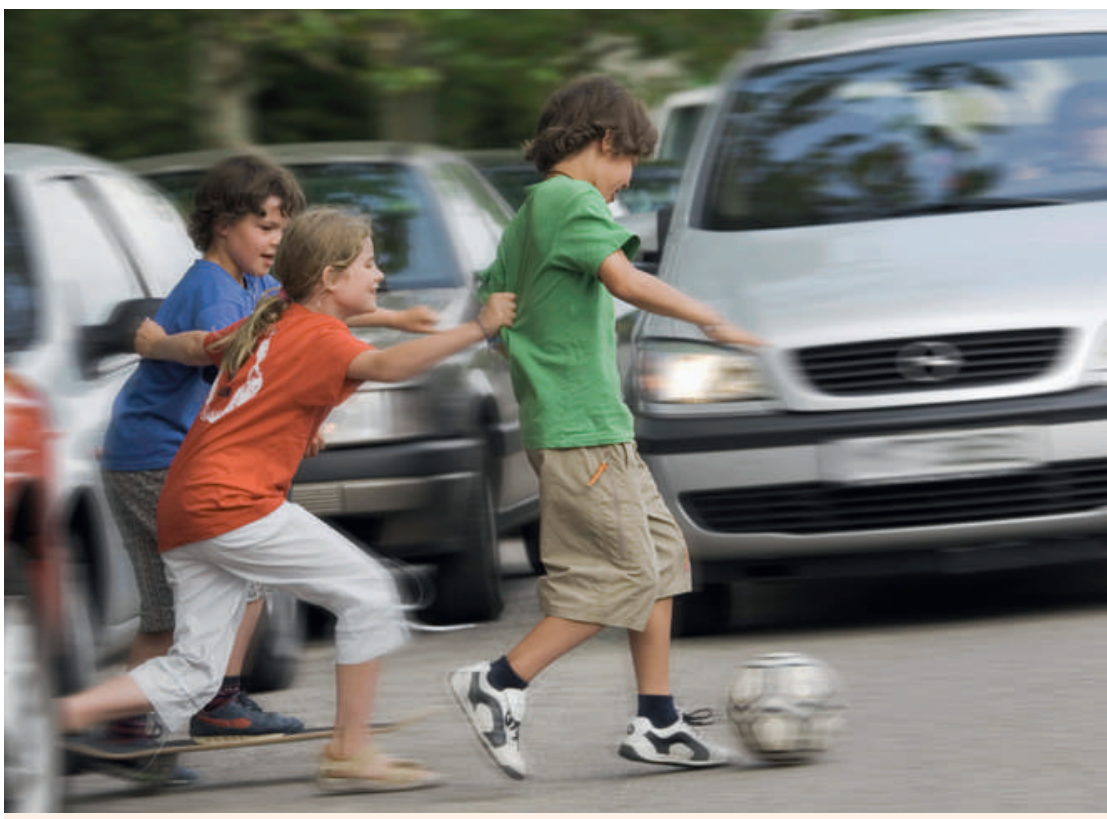

Kommt in der Schweiz ein Kind bei einem Unfall zu Schaden, greift unabhängig von der Schuldfrage die No Fault Compensation - die Haftpflichtversicherung des Fahrers zahlt.
Fahrer nicht schuld ist. Anders in der Medizin, hier gilt zwar für die öffentlichen Spitäler die Kausalhaftung. Aber es müssen dennoch ein objektiver Fehler, ein Schaden sowie ein Zusammenhang zwischen Fehler und Schaden nachgewiesen werden [2]. Der Patient muss dem Arzt oder dem Spital einen Fehler nachweisen. Das führt $\mathrm{zu}$ viel Streit, beschäftigt Gerichte und Rechtsanwälte. Berechtigte Entschädigungen lassen oft (zu) lange auf sich warten.

\section{No Fault Compensation in der Medizin}

Die FMH hat schon in den 90er Jahren und 2002 Anstrengungen unternommen, die No Fault Compensation einzuführen, aber leider ohne Erfolg [2]. Ein neuer Versuch lohnt sich. Vor allem für die Patienten, denen viel Leid erspart wird. Aber auch für die Ärzte, die nicht mehr in Prozesse involviert werden, ausser bei strafrechtlich relevanten Tatbeständen. Was für eine Erleichterung!

Finanzierungsvorschlag gefällig? Jeder Arzt und jedes Spital in der Schweiz sind haftpflichtversichert. Da kommen bedeutende Prämiengelder zusammen, wahrscheinlich mehr als die Summe, die benötigt wird, um per No Fault Compensation alle Ansprüche der Geschädigten zu erfüllen. Die Haftpflichtprämien fallen auf einen Teil der aktuellen. Und obendrein: Zahllose (Zusatz-)Untersuchungen, die heute nur aus rechtlichen Gründen gemacht werden, entfallen. Eine bedeutende Einsparung für das Gesundheitswesen.

\section{No Fault Compensation: ein Segen}

Die No Fault Compensation in der Medizin ist segensreich. Wird viel des entstandenen Leides mildern. Verhindert und mindert Verbitterung aufseiten von Patientinnen und Patienten sowie Ärztinnen und Ärzten. Und würde die Amerikanisierung unseres medizinischen Haftpflichtrechtes stoppen. Die FMH sollte den Faden zur Einführung der No Fault Compensation in der Medizin wieder aufnehmen und sich durchsetzen. Und sich damit als verdienstlich und auf der Höhe der Zeit erweisen.

\section{Referenzen}

1 No Fault Compensation Corporation in New Zealand. Wikipedia

2 Ich danke Hanspeter Kuhn, Leiter Rechtsdienst der $\mathrm{FMH}$, für seine wertvollen Hinweise und Informationen. 